\title{
OPTIMAL PROPORTIONAL REINSURANCE UNDER TWO CRITERIA: MAXIMIZING THE EXPECTED UTILITY AND MINIMIZING THE VALUE AT RISK
}

\author{
ZHIBIN LIANG $^{\otimes 1}$ and JUNYI GUO ${ }^{2}$
}

(Received 17 May, 2010; revised 24 September, 2010)

\begin{abstract}
We consider the optimal proportional reinsurance from an insurer's point of view to maximize the expected utility and minimize the value at risk. Under the general premium principle, we prove the existence and uniqueness of the optimal strategies and Pareto optimal solution, and give the relationship between the optimal strategies. Furthermore, we study the optimization problem with the variance premium principle. When the total claim sizes are normally distributed, explicit expressions for the optimal strategies and Pareto optimal solution are obtained. Finally, some numerical examples are presented to show the impact of the major model parameters on the optimal results.
\end{abstract}

2000 Mathematics subject classification: primary 91B30; secondary 93E20.

Keywords and phrases: proportional reinsurance, expected utility, value at risk, premium principle, Pareto optimal solution.

\section{Introduction}

In the past few years, optimal reinsurance problems have attracted much interest in the actuarial literature. With reinsurance one reduces the risk insured by the company at the expense of reducing the potential profit. Therefore, the insurer should seek the optimal reinsurance strategy to balance risk and profits. There are many approaches to choosing reinsurance contracts. One of them is to apply the technique of stochastic control theory and the Hamilton-Jacobi-Bellman equation; see, for example, Schmidli [25, 26], Promislow and Young [23], Luo et al. [22], Irgens and Paulsen [15], Liang [19] and Zhang et al. [30]. Another method is based on maximizing stability measured by the variance or other functions, see Kaluszka [16, 17], Kaluszka and Okolewski [18] and the references therein. Since an explicit expression for the ruin probability for the jump-diffusion process is difficult to derive, some of the literature

\footnotetext{
${ }^{1}$ School of Mathematical Sciences, Nanjing Normal University, Jiangsu 210046, PR China; e-mail: liangzhibin111@hotmail.com.

${ }^{2}$ School of Mathematical Sciences, Nankai University, Tianjin 300071, PR China.

(C) Australian Mathematical Society 2010, Serial-fee code 1446-1811/2010\$16.00
} 
focuses on constructing optimal contracts to maximize the adjustment coefficient by the Martingale approach; see, for example, Waters [29], Centeno [5], Hald and Schmidli [13], Liang and Guo [20, 21]. A final approach relies on minimizing tail risk measures, such as value at risk (VaR) and conditional tail expectation (CTE); see, for example, Cai and Tan [3], Cai et al. [4], Bernard and Tian [2].

However, most of the literature above only considers the optimal reinsurance problem with a single criterion, for example, minimizing the risk or maximizing the profit. From the numerical examples of Liang [19], we can see that the optimal reinsurance strategy is very different under different criteria: a strategy which is optimal for one criterion may be unreasonable for another. Therefore, it is very important to have a real optimal reinsurance strategy which maximizes profit while minimizing risk. Most of the optimization problems for balancing risk and profits can be found in financial models, very few of them in insurance risk models. For example, Emmer et al. [9] consider some continuous-time Markowitz-type portfolio problems that consist of maximizing expected terminal wealth under the constraint of an upper bound for the capital at risk. Closed-form explicit solutions are obtained. Basak and Shapiro [1] analyse optimal dynamic portfolio and wealth/consumption policies of utility maximization with VaR constraint. They suggest an alternative risk management model, based on the expectation of a loss, to remedy the shortcomings of VaR. Kaluszka [16, 17] and Kaluszka and Okolewski [18] consider the optimal reinsurance arrangements for balancing risk measured by variance and expected profits, or other functions. Huang [14] designs an optimal insurance contract under a VaR constraint to maximize the expected utility, and derives the optimal insurance contract as a single deductible insurance when the VaR constraint is redundant, or as a double deductible insurance when the VaR constraint is binding. Wang et al. [28] consider the same optimal problem with VaR constraint as in Huang [14], albeit that the criterion is maximizing the expected wealth.

In this paper, from an insurer's point of view, we seek a proportional reinsurance strategy that is optimal under two criteria: maximizing the expected utility and minimizing the VaR. By the technique of multi-criteria optimization theory (see Ehrgott [7]), we discuss the Pareto optimal solution of this two-criteria problem. Under certain conditions, we prove existence and uniqueness of the optimal strategies and Pareto optimal solution for the risk model with the general premium principle. The relationships between the Pareto optimal solution and the other two optimal strategies under a single criterion are given. Under the variance premium principle, we study the two-criteria optimization problem. When the total claim sizes are normally distributed, we derive explicit expressions for the optimal strategies and Pareto optimal solution. Some numerical examples are presented to examine how the optimal results vary in response to changes in the major model parameters.

In Section 2 the model and assumptions are given. In Section 3 we discuss the optimization problem with the general premium principle. In Section 4 we study the optimization problem with the variance premium principle, and give some numerical examples. 


\section{The model}

We consider a (reinsured) risk over a one-year period, so that the insurer's wealth at the end of the year is

$$
S_{I}=u+c-\delta(q)-X_{I},
$$

where $u$ is the insurer's wealth at the start of the year, $c$ is the premium the insurer receives to cover the risk, $\delta(q)$ is the amount of the reinsurance premium, and $X_{I}$ denotes the amount of claims paid by the insurer. Let $X$ be the (aggregate) loss for an insurer. We assume that $X$ is a nonnegative random variable with cumulative distribution function $F_{X}(x)=\operatorname{Pr}\{X \leq x\}$, survival function $S_{X}(x)=1-F_{X}(x)=$ $\operatorname{Pr}\{X>x\}$ and mean $E X>0$. Furthermore, let $X_{R}$ be the random variable for the loss of the reinsurer in the presence of a proportional reinsurance. Then $X_{I}$ and $X_{R}$ are related to $X$ as follows:

$$
X_{I}=q X \quad \text { and } \quad X_{R}=(1-q) X,
$$

where the parameter $q \in[0,1]$ is known as the retention level. Here, we assume that the reinsurance premium $\delta(q)$ is a continuous function, and satisfies the following assumptions.

Assumption A:

$$
\delta^{\prime}(q)<0 \quad \text { and } \quad \delta^{\prime \prime}(q)>0
$$

Assumption B:

$$
-\delta^{\prime}(1)=E X .
$$

Observe that the following principles are included in this class: for positive constants $\Lambda, c, A$,

Variance premium principle: $\delta(q)=E\left(X_{R}\right)+\Lambda \operatorname{Var}\left(X_{R}\right)$,

Quadratic utility principle: $\delta(q)=E\left(X_{R}\right)+c-\left(c^{2}-\operatorname{Var}\left(X_{R}\right)\right)^{1 / 2}$,

Exponential principle: $\delta(q)=A^{-1} \ln E\left[\exp \left(A X_{R}\right)\right]$.

For an overview of premium calculation principles, we refer the reader to Gerber [10], Goovaerts et al. [11], Straub [27], Embrechts et al. [8], Rolski et al. [24] and Dickson [6].

We denote by $T=\delta(q)+q X$ the total risk or total cost of the insurer. The total risk $T$ is captured by two components: the retained loss and the reinsurance premium. The relation $T=\delta(q)+q X$ demonstrates the classic trade-off between the risk retained by the insurer $(q X)$ and the profit transferred to reinsurance $(\delta(q))$. If the retention $q$ is small, then the retained liability to the insurer is expected to be low but at the expense of the higher premium payable to the reinsurer. On the other hand, reducing the loss of the reinsurance premium by raising $q$ exposes the insurer to a potentially large liability. Consequently, determining the optimal level of retention $q$ is important.

Formally, the VaR of a random variable $X$ at a confidence level $1-\alpha, 0<\alpha<1$, is defined as

$$
\operatorname{VaR}_{X}(\alpha)=\inf \{x: \operatorname{Pr}\{X>x\} \leq \alpha\}=\inf \{x: \operatorname{Pr}\{X \leq x\} \geq 1-\alpha\} .
$$


If $X$ has a continuous one-to-one distribution function on [0, $\infty$ ), then

$$
\operatorname{VaR}_{X}(\alpha)=S_{X}^{-1}(\alpha)=F_{X}^{-1}(1-\alpha),
$$

where $S_{X}^{-1}$ and $F_{X}^{-1}$ are the inverse functions of $S_{X}$ and $F_{X}$, respectively. From Cai and Tan [3] and the definition of VaR, it is not difficult to derive

$$
\operatorname{VaR}_{T}(q, \alpha)=\operatorname{VaR}_{X_{I}}(q, \alpha)+\delta(q),
$$

where

$$
\operatorname{VaR}_{X_{I}}(q, \alpha)=q \operatorname{VaR}_{X}(\alpha)=q S_{X}^{-1}(\alpha)
$$

Then

$$
\operatorname{VaR}_{T}(q, \alpha)=q S_{X}^{-1}(\alpha)+\delta(q) .
$$

In addition, we suppose that the investor has an exponential utility function

$$
U(x)=\lambda-\frac{\gamma}{\beta} e^{-\beta x},
$$

where $\gamma>0$ and $\beta>0$. This utility has constant absolute risk aversion parameter $\beta$, as can be seen from the fact that $-U^{\prime \prime}(x) / U^{\prime}(x)=\beta$. Such utility functions play a prominent role in insurance mathematics and actuarial practice, since they are the only utility functions under which the principle of "zero utility" gives a fair premium that is independent of the level of reserves of an insurance company.

From an insurer's point of view, a prudent risk management strategy is to ensure that the risk measures associated with $T$ are as small as possible. At the same time, the insurer wants the expected utility of their wealth to be as large as possible. This motivates us to consider the following three optimization problems:

Problem A:

$$
\min _{q \in[0,1]}\left\{\operatorname{VaR}_{T}(q, \alpha)\right\}
$$

Problem B:

$$
\max _{q \in[0,1]}\left\{E U\left(S_{I}\right)\right\}
$$

Problem C:

$$
\min _{q \in[0,1]}\left\{\operatorname{VaR}_{T}(q, \alpha),-E U\left(S_{I}\right)\right\} .
$$

Using the exponential utility function, we can derive the expected utility of $S_{I}$ :

$$
E U\left(S_{I}\right)=\lambda-\frac{\gamma}{\beta} e^{-\beta(u+c)} \cdot e^{\beta \delta(q)+\ln M_{X}(\beta q)},
$$

where the moment generating function is $M_{X}(t)=E\left(e^{X t}\right)$. We see that maximizing $E U\left(S_{I}\right)$ is equivalent to minimizing $\beta \delta(q)+\ln M_{X}(\beta q)$. Then Problems B and $\mathrm{C}$ can be rewritten as:

Problem B':

$$
\min _{q \in[0,1]}\left\{\beta \delta(q)+\ln M_{X}(\beta q)\right\}
$$


Problem $\mathrm{C}^{\prime}$ :

$$
\min _{q \in[0,1]}\left\{q S_{X}^{-1}(\alpha)+\delta(q), \beta \delta(q)+\ln M_{X}(\beta q)\right\}
$$

Problem $\mathrm{C}^{\prime}$ is a multi-objective optimization problem and may be converted into a single-objective problem by putting weights on the different objectives. Thus the following function is to be minimized:

$$
f(q)=\lambda_{1}\left[q S_{X}^{-1}(\alpha)+\delta(q)\right]+\lambda_{2}\left[\beta \delta(q)+\ln M_{X}(\beta q)\right]
$$

where $\lambda_{1}, \lambda_{2} \in(0,1), \lambda_{1}+\lambda_{2}=1$, represent the weights.

By the multiple-criteria optimization theory (see Ehrgott [7]), we see that, if we can find the unique positive optimal strategy $q^{*} \in[0,1]$ which minimizes the function $f(q)$, then this optimal strategy is the properly Pareto optimal solution of Problem $\mathrm{C}^{\prime}$ and, consequently, the properly Pareto optimal solution of Problem C. In addition, to guarantee the existence of the optimal strategy, we assume the following condition:

Condition A:

$$
-\delta^{\prime}(0)>S_{X}^{-1}(\alpha)>-\delta^{\prime}(1)
$$

REMARK 2.1. We assume throughout this paper that $X$ has a continuous one-to-one distribution function on $(0, \infty)$ with a possible jump at 0 , and that $S_{X}^{-1}(x)$ exists for $0<x<S_{X}(0)$. Moreover, we write $S_{X}^{-1}(0)=\infty$ and $S_{X}^{-1}(x)=0$ for $S_{X}(0) \leq x \leq 1$. We also enforce the condition $0<\alpha<S_{X}(0)$, otherwise for $\alpha \geq S_{X}(0)$ we have a trivial case as $\operatorname{VaR}_{X}(\alpha)=0$ and $\operatorname{VaR}_{X_{I}}(q, \alpha)=0$. Note that $S_{X}(0)=1$ when the distribution function of $X$ is continuous at 0 .

REMARK 2.2. To guarantee the existence of $M_{X}(t)$, the (aggregate) loss distribution should have an exponentially decreasing tail $S_{X}(x)$. This means that the tail $S_{X}(x)$ satisfies $S_{X}(x)=o\left(e^{-s x}\right)$ for some $s>0$. It is, for instance, fulfilled if there is an $r_{\infty}>0$ such that $\lim _{r \uparrow r_{\infty}} M_{X}(r)=\infty$. This is exactly the condition needed for the Cramér-Lundberg approximation in the classical risk model (see, for instance, Gerber [10] or Grandell [12]).

\section{The optimal strategy with the general premium principle}

We consider the optimal strategies in the above three problems with the general premium principle.

3.1. Existence and uniqueness of the optimal strategies Differentiating $f(q)$ (see (2.1)) with respect to $q$ and simplifying yields

$$
\frac{\partial f}{\partial q}=\left(\lambda_{1}+\lambda_{2} \beta\right) \delta^{\prime}(q)+\lambda_{2} \cdot \frac{M_{X}^{\prime}(\beta q)}{M_{X}(\beta q)}+\lambda_{1} S_{X}^{-1}(\alpha),
$$

where

$$
M_{X}^{\prime}(\beta q)=E\left(\beta X e^{\beta q X}\right)
$$


To find $q$ such that $f(q)$ is minimized to find the unique solution of the equation $\partial f / \partial q=0$, or equivalently,

$$
\frac{M_{X}^{\prime}(\beta q)}{M_{X}(\beta q)}=-\beta \delta^{\prime}(q)-\frac{\lambda_{1}}{\lambda_{2}}\left[\delta^{\prime}(q)+S_{X}^{-1}(\alpha)\right] .
$$

By the same method, we derive another two equations which the optimal strategies in Problems B' and A satisfy:

$$
\frac{M_{X}^{\prime}(\beta q)}{M_{X}(\beta q)}=-\beta \delta^{\prime}(q)
$$

and

$$
\delta^{\prime}(q)+S_{X}^{-1}(\alpha)=0 .
$$

In the following theorem, we verify that the above equations for $q$ have a unique positive solution.

THEOREM 3.1. Under Condition A, each of the Equations (3.2), (3.3) and (3.4) has a unique positive solution.

To verify the theorem, we first prove the following lemmas which play key roles in this paper.

LEMMA 3.2. The function $\ln M_{X}(r)$ is strictly increasing and convex.

PROOF. Let $g(r)=\ln M_{X}(r)$. Then

$$
g^{\prime}(r)=\frac{M_{X}^{\prime}(r)}{M_{X}(r)}=\frac{E\left(X e^{r X}\right)}{E\left(e^{r X}\right)}
$$

and

$$
g^{\prime \prime}(r)=\frac{M_{X}^{\prime \prime}(r) M_{X}(r)-\left[M_{X}^{\prime}(r)\right]^{2}}{\left[M_{X}(r)\right]^{2}} .
$$

By the Cauchy-Schwarz inequality,

$$
E\left(X^{2} e^{r X}\right) E\left(e^{r X}\right)-E\left(X e^{r X}\right)^{2}>0 .
$$

Since

$$
M_{X}^{\prime \prime}(r) M_{X}(r)-M_{X}^{\prime}(r)^{2}=E\left(X^{2} e^{r X}\right) E\left(e^{r X}\right)-E\left(X e^{r X}\right)^{2},
$$

it follows that $g^{\prime \prime}(r)>0$ for all $r>0$. It is easy to see that $g^{\prime}(r)>0$ for all $r>0$.

From Lemma 3.2, we obtain the following result.

LEMMA 3.3. The function $M_{X}^{\prime}(r) / M_{X}(r)$ is strictly increasing and continuous.

We can now prove the theorem.

Proof of Theorem 3.1. Let

$$
g_{1}(q)=\frac{M_{X}^{\prime}(\beta q)}{M_{X}(\beta q)}
$$


and

$$
f_{1}(q)=-\beta \delta^{\prime}(q)-\frac{\lambda_{1}}{\lambda_{2}}\left[\delta^{\prime}(q)+S_{X}^{-1}(\alpha)\right] .
$$

Then

$$
\begin{aligned}
& f_{1}(0)=-\left(\beta+\frac{\lambda_{1}}{\lambda_{2}}\right) \delta^{\prime}(0)-\frac{\lambda_{1}}{\lambda_{2}} S_{X}^{-1}(\alpha), \\
& f_{1}(1)=-\left(\beta+\frac{\lambda_{1}}{\lambda_{2}}\right) \delta^{\prime}(1)-\frac{\lambda_{1}}{\lambda_{2}} S_{X}^{-1}(\alpha),
\end{aligned}
$$

and

$$
g_{1}(0)=\beta E X, \quad g_{1}(1)=\frac{E\left(\beta X e^{\beta X}\right)}{E\left(e^{\beta X}\right)} .
$$

By Condition A and Assumption B,

$$
f_{1}(0)>g_{1}(0), \quad f_{1}(1)<g_{1}(0) .
$$

From Lemma 3.3, we know that $g_{1}(q)$ is a strictly increasing continuous function, so we have $g_{1}(1)>g_{1}(0)$, and therefore, $g_{1}(1)>f_{1}(1)$.

Since $f_{1}(0)>g_{1}(0)$ and $f_{1}(1)<g_{1}(1)$, by the monotonicity and continuity of $f_{1}(q)$ and $g_{1}(q)$, we see that $g_{1}(q)$ and $f_{1}(q)$ have a unique point of intersection at some $0<q_{3}^{*}<1$. Thus Equation (3.2) has a unique positive solution.

Now consider (3.3). Let $f_{2}(q)=-\beta \delta^{\prime}(q)$. We obtain

$$
f_{2}(0)=-\beta \delta^{\prime}(0) \quad \text { and } \quad f_{2}(1)=-\beta \delta^{\prime}(1) .
$$

By Assumptions A and B, and the monotonicity of $g_{1}(q)$ we derive

$$
f_{2}(0)>g_{1}(0) \text { and } f_{2}(1)<g_{1}(1)
$$

Again by the monotonicity and continuity of $f_{2}(q)$ and $g_{1}(q)$, we see that $g_{1}(q)$ and $f_{2}(q)$ have a unique point of intersection at some $0<q_{2}^{*}<1$. Thus (3.3) has a unique positive solution.

Finally, we discuss (3.4). Since $\delta^{\prime}(q)$ is a strictly increasing continuous function such that $-\delta^{\prime}(0)>S_{X}^{-1}(\alpha)>-\delta^{\prime}(1)$, there exists a unique positive value $q_{1}^{*} \in(0,1)$ which satisfies $S_{X}^{-1}(\alpha)=-\delta^{\prime}\left(q_{1}^{*}\right)$.

By Theorem 3.1, we derive the following result.

THEOREM 3.4. Under Condition A, let $q_{1}^{*}, q_{2}^{*}$ and $q_{3}^{*}$ be respectively the unique positive roots of (3.4), (3.3) and (3.2). Then $q_{1}^{*}$ and $q_{2}^{*}$ are respectively the optimal strategies for Problems $A$ and $B$, and $q_{3}^{*}$ is the Pareto optimal solution for Problem $C$. 


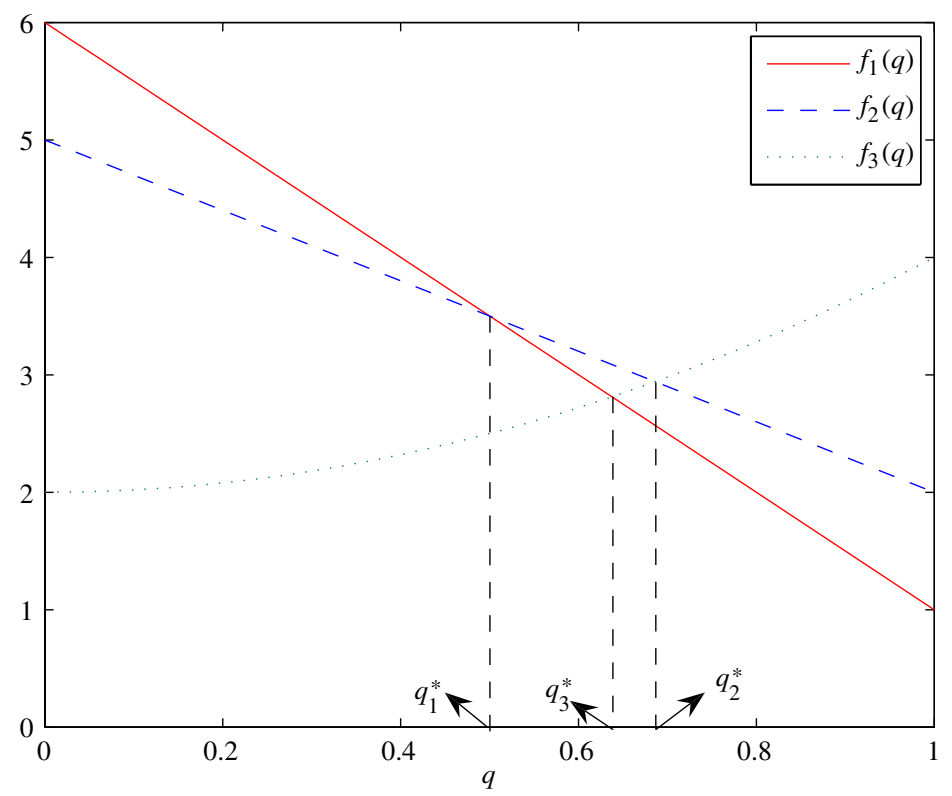

FIGURE 1. Functions $f_{1}(q), f_{2}(q)$ and $f_{3}(q)$ giving $q_{1}^{*}<q_{3}^{*}<q_{2}^{*}$.

3.2. Relationship between the three optimal strategies The following results give the relationship between the three optimal strategies.

THEOREM 3.5. Let $q_{1}^{*}, q_{2}^{*}$ and $q_{3}^{*}$ be the optimal strategies mentioned in Theorem 3.4, and let $M=\max \left(q_{1}^{*}, q_{2}^{*}\right), N=\min \left(q_{1}^{*}, q_{2}^{*}\right)$. Then $q_{3}^{*} \in(N, M)$.

PROOF. Let

$$
f_{1}(q)=-\beta \delta^{\prime}(q)-\frac{\lambda_{1}}{\lambda_{2}}\left[\delta^{\prime}(q)+S_{X}^{-1}(\alpha)\right], \quad f_{2}(q)=-\beta \delta^{\prime}(q), \quad f_{3}(q)=\frac{M_{X}^{\prime}(\beta q)}{M_{X}(\beta q)} .
$$

We see that $q_{1}^{*}$ is the unique solution of the equation $f_{1}(q)=f_{2}(q), q_{2}^{*}$ is the unique solution of $f_{2}(q)=f_{3}(q)$, and $q_{3}^{*}$ is the unique solution of $f_{1}(q)=f_{3}(q)$.

If $q_{1}^{*}<q_{2}^{*}$, to prove that $q_{1}^{*}<q_{3}^{*}<q_{2}^{*}$, it is sufficient to prove that $f_{2}(q)>f_{1}(q)$ for all $q>q_{1}^{*}$; see Figure 1. Note that

$$
f_{2}(q)-f_{1}(q)=\frac{\lambda_{1}}{\lambda_{2}}\left(\delta^{\prime}(q)+S_{X}^{-1}(\alpha)\right) .
$$

Since $\delta^{\prime}(q)+S_{X}^{-1}(\alpha)$ is an increasing function of $q$, when $q>q_{1}^{*}$,

$$
\delta^{\prime}(q)+S_{X}^{-1}(\alpha)>\delta^{\prime}\left(q_{1}^{*}\right)+S_{X}^{-1}(\alpha)=0,
$$

and thus we derive $f_{2}(q)>f_{1}(q)$, for all $q>q_{1}^{*}$. 


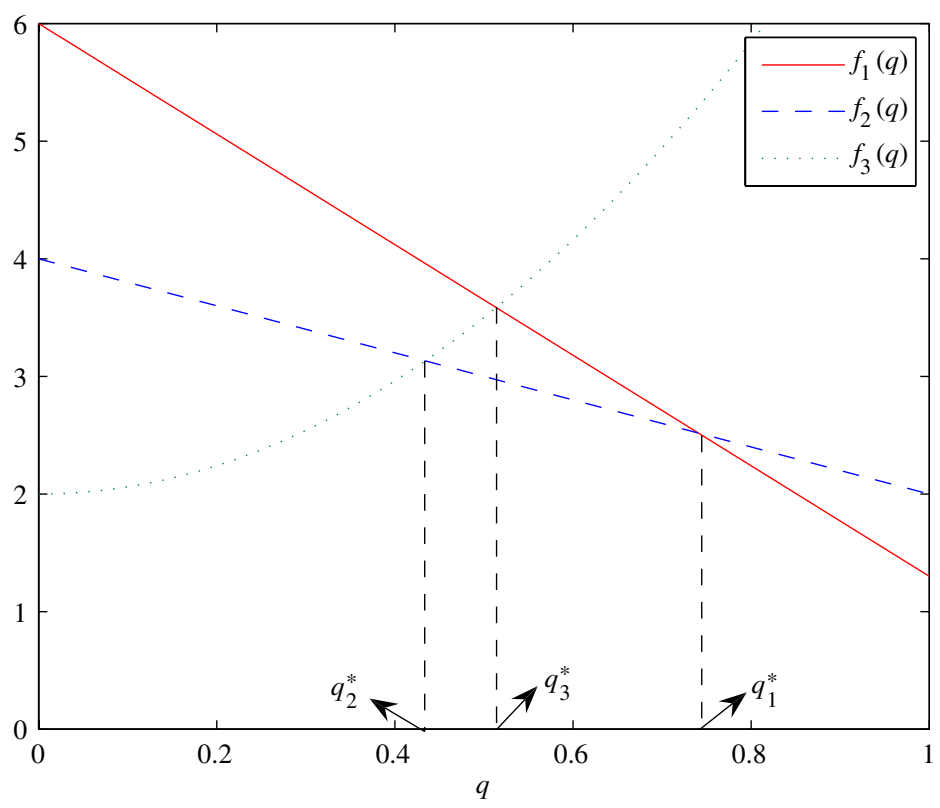

FIGURE 2. Functions $f_{1}(q), f_{2}(q)$ and $f_{3}(q)$ giving $q_{2}^{*}<q_{3}^{*}<q_{1}^{*}$.

If $q_{1}^{*}>q_{2}^{*}$, to prove that $q_{2}^{*}<q_{3}^{*}<q_{1}^{*}$, it is sufficient to prove that $f_{1}(q)>f_{2}(q)$ for all $q<q_{1}^{*}$; see Figure 2. Note that

$$
f_{1}(q)-f_{2}(q)=-\frac{\lambda_{1}}{\lambda_{2}}\left(\delta^{\prime}(q)+S_{X}^{-1}(\alpha)\right) .
$$

Since $\delta^{\prime}(q)+S_{X}^{-1}(\alpha)$ is an increasing function of $q$, when $q<q_{1}^{*}$, we have $\delta^{\prime}(q)+S_{X}^{-1}(\alpha)<\delta^{\prime}\left(q_{1}^{*}\right)+S_{X}^{-1}(\alpha)=0$, and thus we derive $f_{1}(q)>f_{2}(q)$, for all $q<q_{1}^{*}$.

In addition, by (2.1) and (3.1), we derive the following theorem.

THEOREM 3.6. When $\lambda_{1} \rightarrow 0$, we have $q_{3}^{*} \rightarrow q_{2}^{*}$, and when $\lambda_{2} \rightarrow 0$, we have $q_{3}^{*} \rightarrow q_{1}^{*}$.

REMARK 3.7. The results above coincide with the real world. Since the insurer wants to balance the $\mathrm{VaR}$ and expected utility, that is, minimize the $\mathrm{VaR}$ and maximize the expected utility, the corresponding optimal strategy must take a value in $(N, M)$. Furthermore, its value depends on the weights. For instance, $\lambda_{1}<1 / 2$ means that the insurer pays more attention to expected utility, and the corresponding optimal strategy becomes closer to $q_{2}^{*}$ than when $\lambda_{1} \geq 1 / 2$, and vice versa. 


\section{The optimal strategy with the variance premium principle}

In this section, we assume that the reinsurance premium is calculated according to the variance principle, that is, $\delta(q)=E\left(X_{R}\right)+\Lambda \operatorname{Var}\left(X_{R}\right)$, and the total loss $X$ is normally distributed with parameters $\mu$ and $\sigma^{2}$, denoted by $X \sim N\left(\mu, \sigma^{2}\right)$. Then

$$
\begin{aligned}
\delta(q) & =(1-q) \mu+\Lambda(1-q)^{2} \sigma^{2}, \\
\delta^{\prime}(q) & =-\mu-2 \Lambda(1-q) \sigma^{2}, \quad \delta^{\prime \prime}(q)=2 \Lambda \sigma^{2}, \\
S_{X}^{-1}(\alpha) & =\mu+\sigma u_{\alpha},
\end{aligned}
$$

where $u_{\alpha}$ is the $100(1-\alpha)$ th percentile of the standard normal distribution. Here Condition A becomes:

Condition $\mathrm{A}^{\prime}$ :

$$
0<u_{\alpha}<2 \Lambda \sigma
$$

Lemma 4.1. Assume that $X \sim N\left(\mu, \sigma^{2}\right)$. Under Condition $A^{\prime}$, the optimal proportional reinsurance to minimize $\operatorname{VaR}_{T}(\alpha, q)$ is

$$
q_{1}^{*}=1-\frac{u_{\alpha}}{2 \Lambda \sigma} \text {. }
$$

LeMma 4.2. Assume that $X \sim N\left(\mu, \sigma^{2}\right)$. The optimal proportional reinsurance to maximize the expected utility $E U\left(S_{I}\right)$ is

$$
q_{2}^{*}=\frac{2 \Lambda}{\beta+2 \Lambda} .
$$

Lemma 4.3. Assume that $X \sim N\left(\mu, \sigma^{2}\right)$. Under Condition $A^{\prime}$, the optimal proportional reinsurance to minimize $f(q)$ in (2.1) is

$$
q_{3}^{*}=\frac{2 \Lambda \sigma \beta+\left(\lambda_{1} / \lambda_{2}\right) 2 \Lambda \sigma-\left(\lambda_{1} / \lambda_{2}\right) u_{\alpha}}{\sigma \beta^{2}+2 \Lambda \sigma \beta+\left(\lambda_{1} / \lambda_{2}\right) 2 \Lambda \sigma} .
$$

Lemmas 4.1, 4.2 and 4.3 are natural consequences of Theorem 3.4, so we omit the proofs.

Comparing $q_{1}^{*}$ and $q_{2}^{*}$ above yields the following result.

Proposition 4.4. Assume that $X \sim N\left(\mu, \sigma^{2}\right)$. Under Condition $A^{\prime}$, we have:

(i) if $\beta<2 \Lambda u_{\alpha} /\left(2 \Lambda \sigma-u_{\alpha}\right)$, then $q_{2}^{*}>q_{1}^{*}$ and $q_{1}^{*}<q_{3}^{*}<q_{2}^{*}$;

(ii) if $\beta>2 \Lambda u_{\alpha} /\left(2 \Lambda \sigma-u_{\alpha}\right)$, then $q_{2}^{*}<q_{1}^{*}$ and $q_{2}^{*}<q_{3}^{*}<q_{1}^{*}$.

REMARK 4.5. Here $\beta$ is the constant absolute risk aversion parameter of the utility function. A larger value of $\beta$ means more risk aversion for the decision maker. Let $\beta_{0}=2 \Lambda u_{\alpha} /\left(2 \Lambda \sigma-u_{\alpha}\right)$. Then, under the criterion of maximizing the expected utility, we see that when $\beta>\beta_{0}$, the decision maker has larger risk aversion and wants to transfer more risk to the reinsurer. Conversely, when $\beta<\beta_{0}$, the decision maker has smaller risk aversion and wants to retain more risk.

We give some numerical examples. For notational convenience, we denote by $m$ the ratio of $\lambda_{1}$ to $\lambda_{2}$, that is, $m=\lambda_{1} / \lambda_{2}$. 


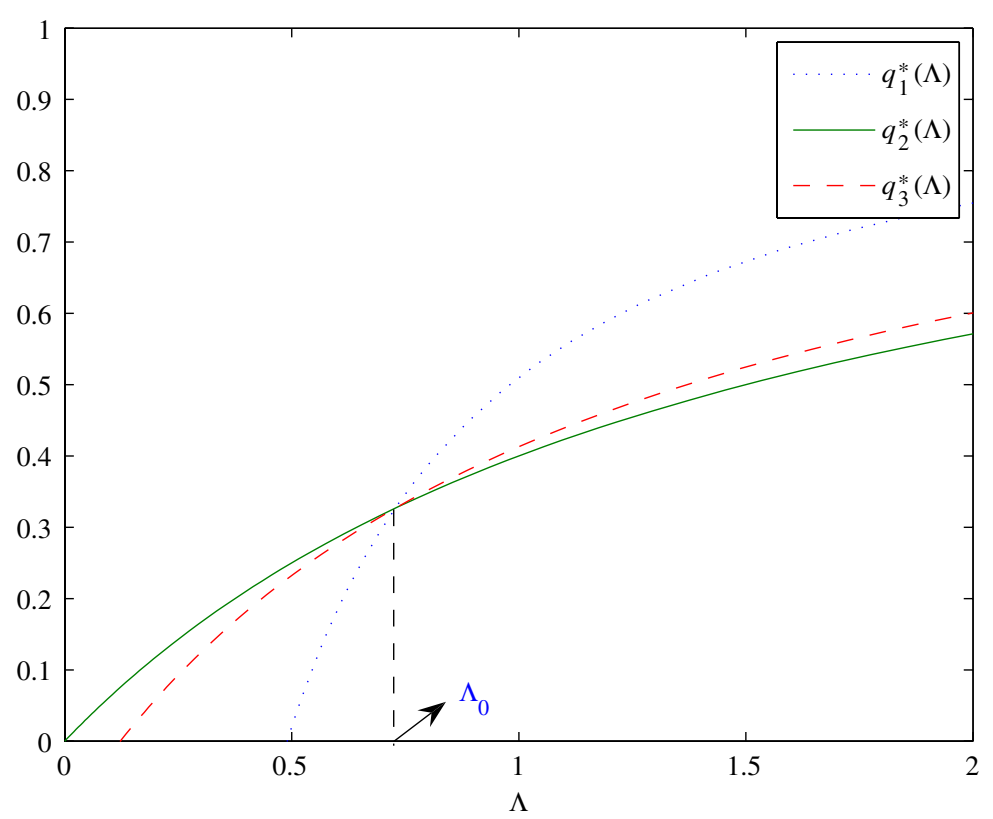

FIGURE 3. The effect of $\Lambda$ on $q_{1}^{*}, q_{2}^{*}$ and $q_{3}^{*}$.

EXAmple 4.6. Assume that $X \sim N(3,4), \alpha=0.05, m=1$. The results are shown in Figures 3 and 4.

From Figure $3(\beta=3)$, we see that the optimal strategies increase as $\Lambda$ increases, which means that a more expensive reinsurance premium yields a greater retention level. Moreover, there exists a $\Lambda_{0}$ such that when $\Lambda<\Lambda_{0}$, we have $q_{1}^{*}<q_{3}^{*}<q_{2}^{*}$, whereas when $\Lambda>\Lambda_{0}$, we have $q_{2}^{*}<q_{3}^{*}<q_{1}^{*}$. From Figure $4(\Lambda=1)$ we can see that there exists a $\beta_{0}$ such that when $\beta<\beta_{0}$, we have $q_{1}^{*}<q_{3}^{*}<q_{2}^{*}$, and when $\beta>\beta_{0}$, we have $q_{2}^{*}<q_{3}^{*}<q_{1}^{*}$. These two results are natural consequences of Proposition 4.4.

ExAmple 4.7. Assume that $X \sim N(3,4), \Lambda=1, \beta=1,3$ and $\alpha=0.05,0.0394$, 0.0192. The results are shown in Figure 5.

In this case, we get $1<\beta_{0}=2 \Lambda u_{\alpha} /\left(2 \Lambda \sigma-u_{\alpha}\right)<3$. From case (a) in Figure 5 we find that, when $\beta=1$, the optimal reinsurance strategy decreases when the value of $m$ increases. Whereas when $\beta=3$ (see case (b) in Figure 5), the optimal strategy increases when the value of $m$ increases. From both cases, we also see that a larger value of $\alpha$ will yield a higher retention level of optimal reinsurance. This simply states that as the confidence level decreases, the insurer should retain a greater share of each claim. 


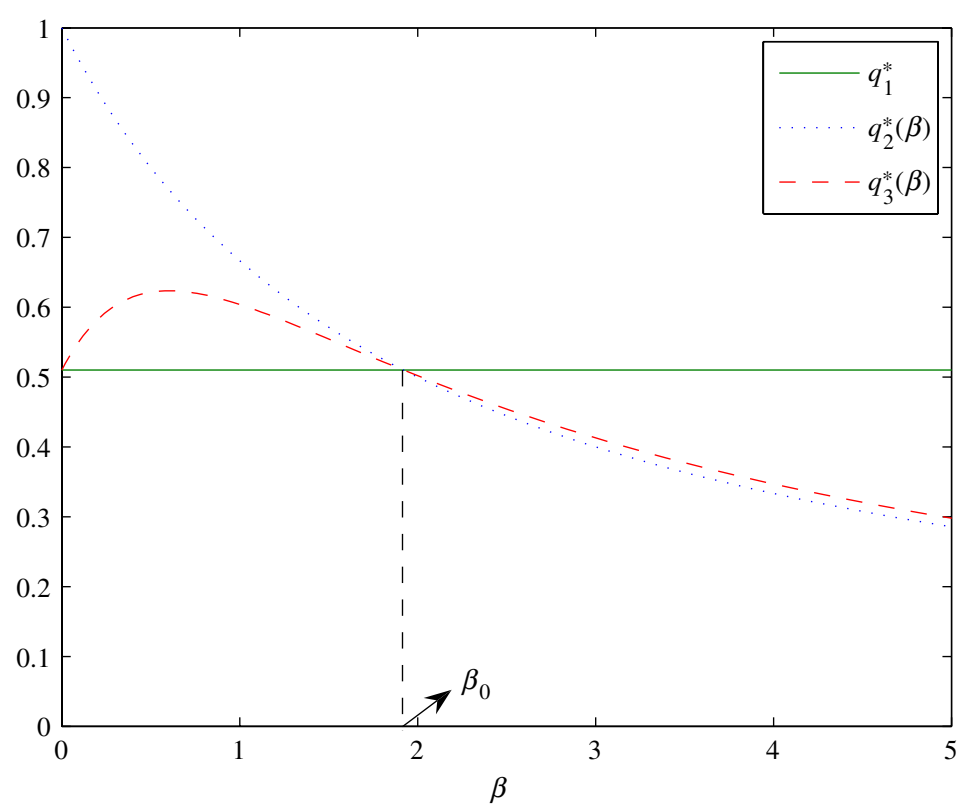

FIGURE 4. The effect of $\beta$ on $q_{1}^{*}, q_{2}^{*}$ and $q_{3}^{*}$.
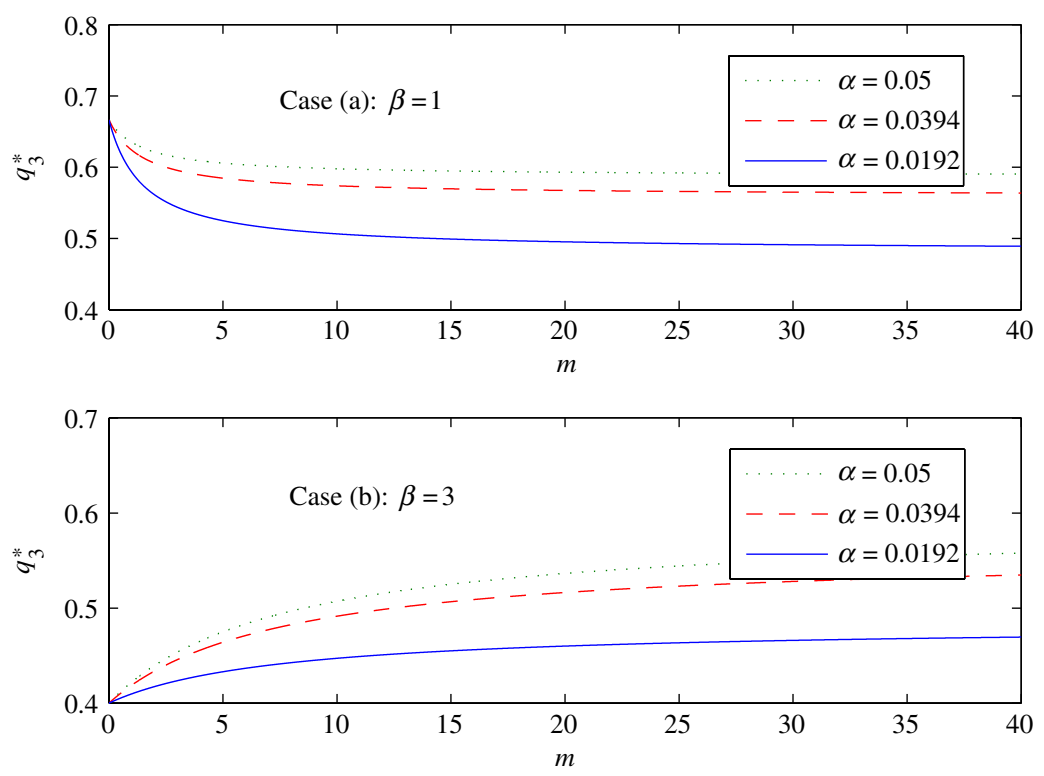

FIGURE 5. The effect of $m$ on $q_{3}^{*}$. 

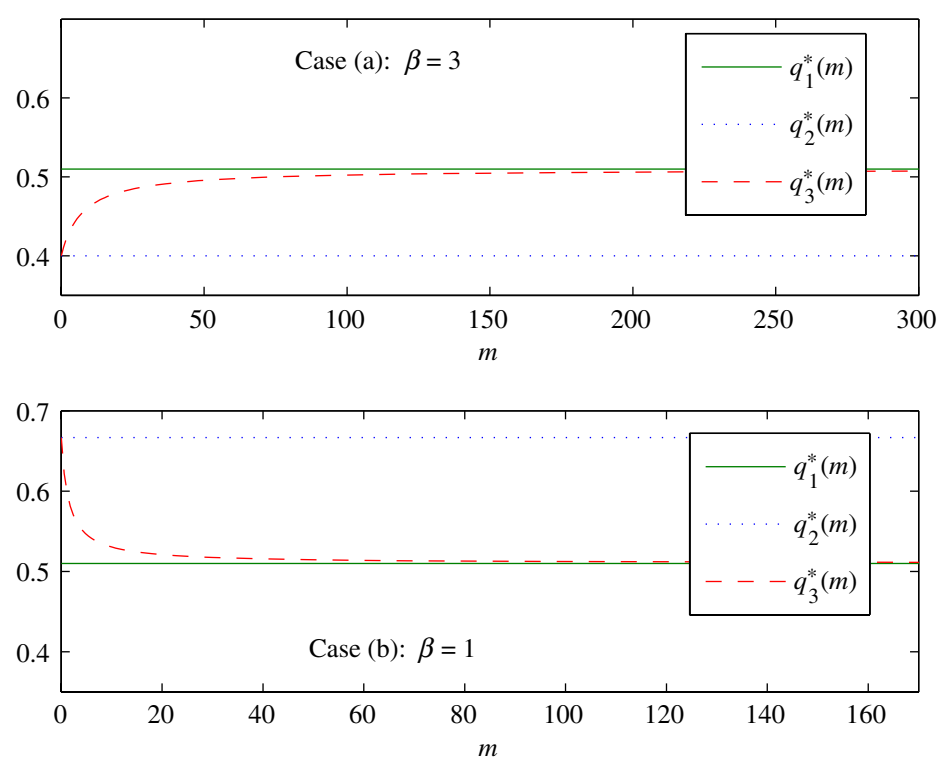

FIGURE 6. The effect of $m$ on $q_{1}^{*}, q_{2}^{*}$ and $q_{3}^{*}$.

EXAmple 4.8. Assume that $X \sim N(3,4), \alpha=0.05, \Lambda=1, \beta=1,3$. The results are shown in Figure 6.

In this case, we get $\beta_{0}=2 \Lambda u_{\alpha} /\left(2 \Lambda \sigma-u_{\alpha}\right)=1.921$. Since $\beta=1<\beta_{0}$ and $\beta=3>\beta_{0}$, we have $q_{1}^{*}<q_{3}^{*}<q_{2}^{*}$ when $\beta=1$ and $q_{2}^{*}<q_{3}^{*}<q_{1}^{*}$ when $\beta=3$. This is again a natural consequence of Proposition 4.4. Furthermore, we find that when $\lambda_{1} \rightarrow 0$, we have $q_{3}^{*} \rightarrow q_{2}^{*}$, and when $\lambda_{2} \rightarrow 0, q_{3}^{*} \rightarrow q_{1}^{*}$, which is a direct result of Theorem 3.6.

\section{Conclusion}

We briefly summarize the main results of this paper. From an insurer's point of view, the two-criteria optimization problem of proportional reinsurance is considered. We seek a proportional reinsurance strategy which is optimal to maximize the expected utility as well as minimize the VaR. Using the technique of multi-criteria optimization theory, we discuss the Pareto optimal solution. Under the general premium principle, we prove the existence and uniqueness of the optimal strategies and Pareto optimal solution, and give the relationship between the optimal strategies. Furthermore, we study the optimization problem with the variance premium principle. When the total claim sizes are normally distributed, we derive explicit expressions not only for the optimal strategies but also for the Pareto optimal solution. Numerical examples are presented to examine how the optimal results vary in response to changes in the major model parameters. We conclude that to balance risk and profit, a reasonable or acceptable reinsurance strategy should take a value in $(N, M)$ (see Theorem 3.5). 


\section{Acknowledgements}

The authors would like to thank the anonymous referees for their careful reading and helpful comments on an earlier version of this paper, which led to a considerable improvement of the presentation of the work. This work was supported by the National Natural Science Foundation of China (Grant No. 10701082) and the Natural Science Foundation of the Jiangsu Higher Education Institutions of China (Grant No. 09KJB110004).

\section{References}

[1] S. Basak and A. Shapiro, "Value-at-risk-based risk management: optimal policies and asset prices", Rev. Financ. Stud. 14 (2001) 371-405.

[2] C. Bernard and W. Tian, "Optimal reinsurance arrangements under tail risk measures", J. Risk Insurance 76 (2009) 709-725.

[3] J. Cai and K. Tan, "Optimal retention for a stop-loss reinsurance under the VaR and CTE risk measures", Astin Bull. 37 (2007) 93-112.

[4] J. Cai, K. Tan, C. Weng and Y. Zhang, "Optimal reinsurance under VaR and CTE risk measures", Insurance Math. Econom. 43 (2008) 85-196.

[5] M. L. Centeno, "Measuring the effects of reinsurance by the adjustment coefficient", Insurance Math. Econom. 5 (1986) 169-182.

[6] D. C. M. Dickson, Insurance risk and ruin (Cambridge University Press, Cambridge, 2004).

[7] M. Ehrgott, Multicriteria optimization (Springer, Berlin, 2000).

[8] P. Embrechts, C. Klüppelberg and T. Mikosch, Modelling extremal events (Springer, Berlin, 1997).

[9] S. Emmer, C. Klüppelberg and R. Korn, "Optimal portfolios with bounded capital at risk", Math. Finance 11 (2001) 365-384.

[10] H. Gerber, An introduction to mathematical risk theory (S. S. Huebner Foundation for Insurance Education, Wharton School, University of Pennsylvania, Philadelphia, 1979).

[11] M. Goovaerts, F. De Vylder and J. Haezendonck, Insurance premiums (North-Holland, Amsterdam, 1984).

[12] J. Grandell, Aspects of risk theory (Springer, New York, 1991).

[13] M. Hald and H. Schmidli, "On the maximization of the adjustment coefficient under proportional reinsurance", Astin Bull. 34 (2004) 75-83.

[14] H. H. Huang, "Optimal insurance contract under a value-at-risk constraint", Geneva Risk Insurance Rev. 31 (2006) 91-110.

[15] C. Irgens and J. Paulsen, "Optimal control of risk exposure, reinsurance and investments for insurance portfolios", Insurance Math. Econom. 35 (2004) 21-51.

[16] M. Kaluszka, "Optimal reinsurance under mean-variance premium principles", Insurance Math. Econom. 28 (2001) 61-67.

[17] M. Kaluszka, "Mean-variance optimal reinsurance arrangements", Scand. Actuar. J. 1 (2004) 28-41.

[18] M. Kaluszka and A. Okolewski, "An extension of Arrow's result on optimal reinsurance contract", J. Risk Insurance 75 (2008) 275-288.

[19] Z. Liang, "Optimal proportional reinsurance for controlled risk process which is perturbed by diffusion”, Acta Math. Appl. Sin. Engl. Ser. 23 (2007) 477-488.

[20] Z. Liang and J. Guo, "Optimal proportional reinsurance and ruin probability", Stoch. Models 23 (2007) 333-350.

[21] Z. Liang and J. Guo, "Upper bound for ruin probabilities under optimal investment and proportional reinsurance", Appl. Stoch. Models Bus. Ind. 24 (2008) 109-128.

[22] S. Luo, M. Taksar and A. Tsoi, "On reinsurance and investment for large insurance portfolios", Insurance Math. Econom. 42 (2008) 434-444. 
[23] S. D. Promislow and V. R. Young, "Minimizing the probability of ruin when claims follow Brownian motion with drift", N. Am. Actuar. J. 9 (2005) 109-128.

[24] T. Rolski, H. Schmidli, V. Schmidt and J. Teugels, Stochastic processes for insurance and finance (Wiley, Chichester, 1999).

[25] H. Schmidli, "Optimal proportional reinsurance policies in a dynamic setting", Scand. Actuar. J. 1 (2001) 55-68.

[26] H. Schmidli, "On minimizing the ruin probability by investment and reinsurance", Ann. Appl. Probab. 12 (2002) 890-907.

[27] E. Straub, Non-life insurance mathematics (Springer, Berlin, 1988).

[28] C. P. Wang, D. Shyu and H. H. Huang, "Optimal insurance design under value-at-risk framework", Geneva Risk Insurance Rev. 30 (2005) 161-179.

[29] H. Waters, "Excess of loss reinsurance limits", Scand. Actuar. J. 1 (1979) 37-43.

[30] X. Zhang, K. Zhang and X. Yu, "Optimal proportional reinsurance and investment with transaction costs, I: Maximizing the terminal wealth", Insurance Math. Econom. 44 (2009) 473-478. 